\title{
Correspondence between crystal structures and tabletability in nitrofurantoin cocrystals
}

\author{
Venu R. Vangala ${ }^{1}$ \\ ${ }^{1}$ School Of Pharmacy And University Of Bradford, Bradford, United Kingdom \\ E-mail: V.G.R.Vangala@bradford.ac.uk
}

Materials undergo deformation includes elasticity, plasticity, viscoelasticity, brittle fracture, fragmentation or a combination of these upon applying stress. One of the challenges in pharmaceutical tablet manufacturing is the poor tabletability of an active pharmaceutical ingredient (API). Crystal engineering of cocrystals is an emerging approach to tailor physicochemical properties of drug and nutraceutical substances [1]. Studies concerning mechanical properties of cocrystals have only been conducted recently [2], [3]. There is not much attention paid where cocrystallisation deteriorates API tabletability. A thorough understanding of correspondence between crystal structure and mechanical properties is essential in the context of powder compaction.

For this talk, an antibiotic drug nitrofurantoin (NF) and its cocrystals with various coformers, 4-hydroxybenzoic acid (NF4HBA), 4-hydroxybenzamide (NF-4HBAm), 4-aminobenzoic acid (NF-4ABA), 3-aminobenzoic acid (3ABA) and urea (NFUREA) were considered and their compressibility behaviours have been evaluated. Their tableting performance follows the order of NF > NF-4HBA $\approx N F-4 H B A m \approx N F-4 A B A>N F-3 A B A>>$ NF-UREA. This difference in bulk compaction behaviour originates from the dissimilar crystal plasticity and elasticity, which results from unique molecular packing features in the respective lattices. Among cocrystals, NF-UREA presented with poorest tabletability (Figure 1). Interestingly, its crystal packing correlates well with the observed mechanical behaviour meaning zig-zag arrangement of molecules with inter-planar hydrogen bonding, which dramatically prohibits plastic deformation thus poor tabletability. However, pure NF displays superior tabletability because it undergoes plastic deformation more readily compared to the cocrystals due to slip layers in NF are composed of weak C-H...O interactions, which provide additional flexibility for slip. These findings reinforce the role of crystal structures in understanding pharmaceutical properties.

[1] Vangala, V. R. et al. (2012). Cryst. Growth Des. 12, 5925-5938.

[2] Sun, C. C. \& Hou, H. (2008). Cryst. Growth Des. 8, 1575-1579.

[3] Ahmed, H. et al. (2017). Drug Dev. Ind. Pharm. 43, 89-97.

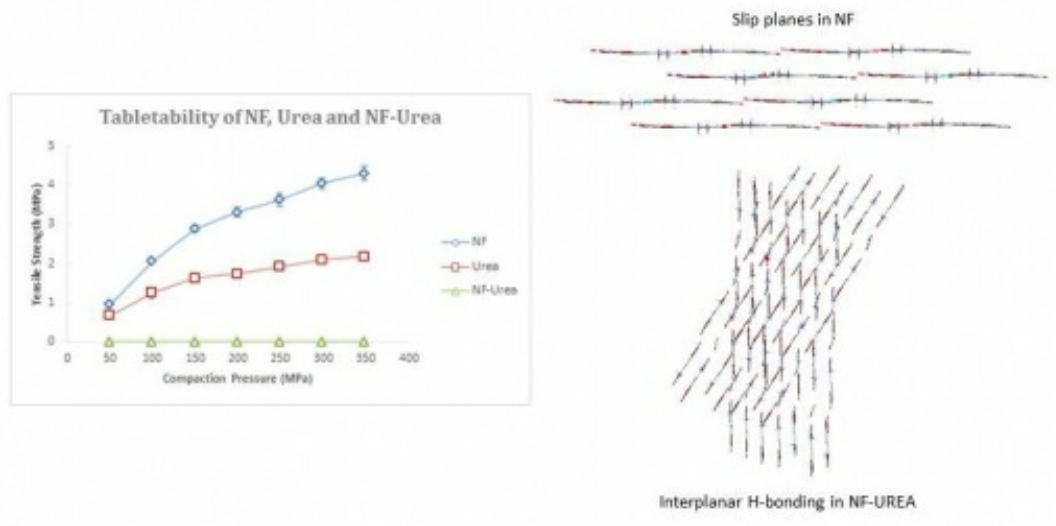

Figure 1. Tabletability of NF, UREA and NF-UREA.

Keywords: crystal engineering, crystal structure vs mechanical properties, pharmaceutical tableting 